\title{
Calling upon all public health mycologists
}

\section{To accompany the country burden papers from 14 countries}

\author{
D. W. Denning ${ }^{1}$
}

Received: 9 January 2017 / Accepted: 9 January 2017 /Published online: 1 February 2017

(C) Springer-Verlag Berlin Heidelberg 2017

Public health mycology is a non-existent discipline currently. Yet, hundreds of epidemiological studies of fungal disease have been and are published, dozens every year. Why the disconnect?

The foundations of public health in infectious diseases are rooted in the transmissibility of severe, sometimes fatal, infections, occurring in the general public. Transmission routes from water (cholera and typhoid), from doctors' and midwives' hands [puerperal fever (and some cases of candidaemia)], from coughing (influenza and whooping cough), from close contact (meningococcal sepsis, measles and rubella), from mosquito bites (malaria and dengue fever) and from sexual contact (hepatitis B and HIV) dominate the learning curricula and efforts in public health. Fungal diseases rarely figure in public health teaching.

So where does that leave fungal disease and public health mycology? The lack of person-to-person transmissibility is a missing link for most fungal infections - acquisition from the environment, from the air or inoculation being the most common modes of acquisition. Cutaneous fungal infections are mostly transmissible from person to person and are estimated to be the fourth most common medical problem after dental caries, tension and migraine headaches [1]. Indeed, nearly a billion people are affected, but they are rarely dramatic or fatal and develop slowly. Tinea capitis is a particular problem of children, with over 200 million affected based on rates in school children in multiple African countries. Some have inflamed, painful lesions that scar. Yet, public health is, today,

D. W. Denning

ddenning@manchester.ac.uk

1 The University of Manchester and Global Action Fund for Fungal Infections, Manchester, UK unmoved - unlike the public health authorities handling early immigrants to Israel or in Victorian England [2]. Several skin and nail infections may herald progressive HIV infection before the onset of AIDS. Tinea pedis or athlete's foot is associated with lower leg cellulitis, a common reason for hospital admission. And subcutaneous infection such as mycetoma and chromoblastomycosis are severe and life-changing infections. Yet, these issues are not on the public health agenda, with the singular exception of mycetoma, recently listed as a Neglected Tropical Disease by the Word Health Organization.

Aside from dermatophyte infections, the only other personto-person transmissible fungal disease is Pneumocystis pneumonia (PCP). Multiple outbreaks are described, typically in transplant patients [3]. But PCP in normal people is rare, so hardly a driver for public health programmes, outside of HIV infection, itself a major global focus for public health.

So the general lack of transmissibility and, therefore, absence of public health mycology has resulted in no public health voice for the hundreds of millions affected by fungal disease. The current series of papers from 14 countries covering 883 million people from all continents (12.5\% of the global population) is a key step in placing serious fungal diseases on the public health agenda. Overall, about $2 \%$ of each country's population is affected, some more, some less.

While there are some published data from each country, it is always incomplete, of variable quality and robustness, and some data are quite old. Most estimates are themselves based on estimates of underlying disease prevalence or annual incidence and, therefore, subject to double errors. Some would argue that sensitivity analyses should be added, but this adds a veneer of precision that is only occasionally appropriate. Some conditions cannot be reliably estimated from underlying diseases or extrapolated from small series, such as tinea capitis, fungal keratitis and mycetoma, and, so, are missing from many of the papers in this series. 


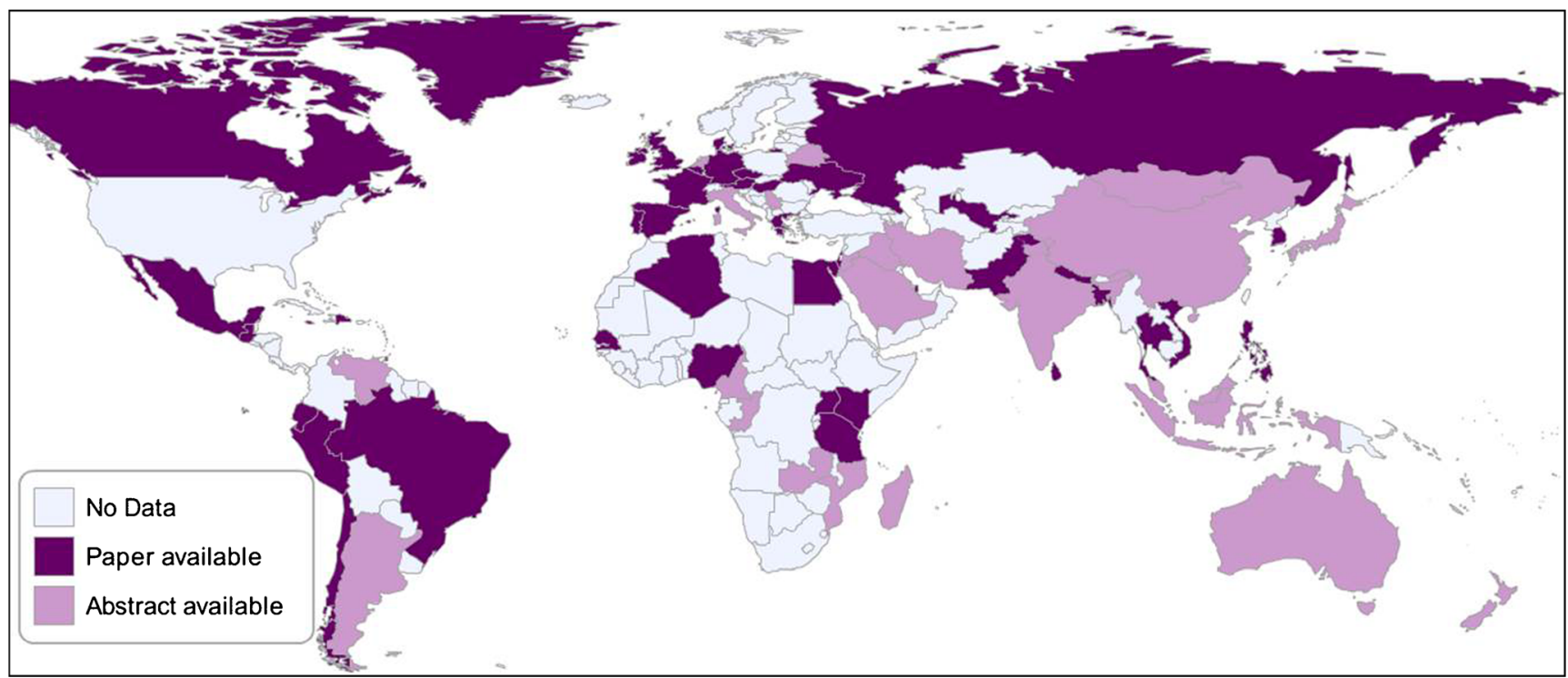

Fig. 1 A world map showing which countries have had fungal disease burden estimates completed, either published (including this series) or simply in abstract form (all abstracts available here: http://life-worldwide.org/media-centre/news)

We have elected to estimate 'serious' fungal infections in a systematic way, as previously described in papers published for another 29 countries (Fig. 1), as well as global estimates of several fungal diseases in multiple countries (http://www. gaffi.org/media/academic-papers/). This should allow international comparison, although where national rates or prevalence estimates are missing, consistent cross-country errors are as likely.

The main objective of each paper is two-fold: (1) to generate approximations and order of magnitude estimates of the key and most common serious fungal disease in each country and (2) to lay out what is known about the burden of these diseases in each country, and what is not. It would be a mistake to criticise any given estimate as being 'wrong' if it is within an order of magnitude of reality. We hope these papers will stimulate much better national (and international) epidemiological studies, supported by improved diagnostics in each country.

\section{References}

1. Vos T, Flaxman AD, Naghavi M, Lozano R, Michaud C, Ezzati M et al (2012) Years lived with disability (YLDs) for 1160 sequelae of 289 diseases and injuries 1990-2010: a systematic analysis for the Global Burden of Disease Study 2010. Lancet 380:2163-2196

2. Homei A, Worboys M (2013) Fungal disease in Britain and the United States 1850-2000. Mycoses and modernity. Palgrave Macmillan, Basingstoke. ISBN-13: 9781137377012

3. Yiannakis EP, Boswell TC (2016) Systematic review of outbreaks of Pneumocystis jirovecii pneumonia: evidence that $P$. jirovecii is a transmissible organism and the implications for healthcare infection control. J Hosp Infect 93:1-8 